QK329

. 578

Studniczka, C

Beiträge zur flora von Süddalmatien 


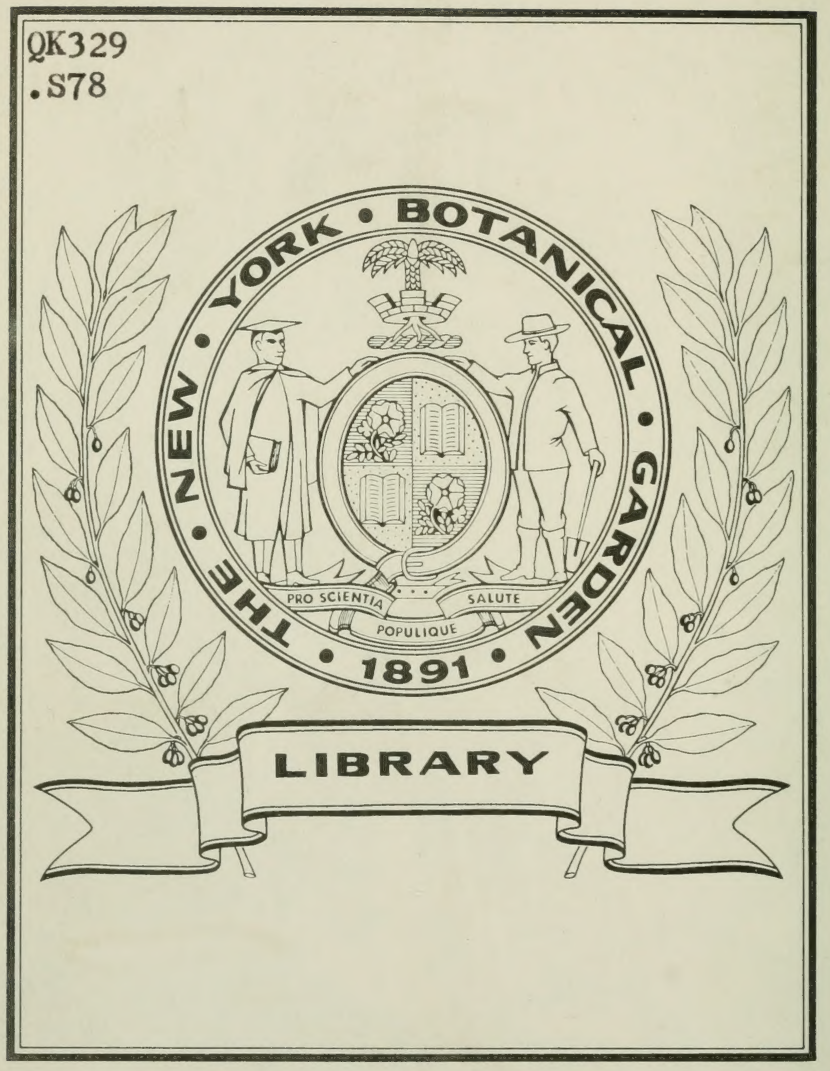



Atudriczka,

Peitrage zur Flore van

Liddalmation.

i. Verhandlangen d.R.R. joolog. botan. Gesellschaft zu wien, XI.B̈d. Fahrg. is go wien 1890 p. $55 \mathrm{pg}$. 



\title{
Beiträge zur Flora von Süddalmatien.
}

\author{
Von
}

\section{Studniezka.}

(Vorgelegt in der Versammlung am 6. Jänner 1890.)

Während meines fast achtjährigen Aufenthaltes in Süddalmatien, besonders in der Bocehe di Cattaro, war es mir möglich, manche neue Standorte meist phanerogamischer Pflanzen zu finden, welche in Visiani's „Flora Dalmatica“ nicht angeführt erscheinen.

Damit diese oft nahe der Küste gelegenen Standorte nicht in Vergessenheit gerathen, erlaube ich mir nachstehendes Verzeichniss der von Trau bis Budua beobachteten Pflanzen hiemit zu veröffentlichen, wobei ich nicht unterlassen kann dankbarst zu bemerken, dass ich in der Bestimmung zahlreicher Pflanzen durch gütige Mittheilungen des Herrn Baurathes J. Freyn als Neuling wesentlich unterstützt wurde.

\section{Equisetaceen.}

Equisetum Telmateja Ehrh. An schattigen Bachrändern bei Topla und Meligne nächst Castelnuovo, auch in der Dobrota und im Zuppathale unterhalb Trinità bei Cattaro (April, Mai).

\section{Polypodiaceen.}

Polypodium vulgare L. In Gebüschen beim Dorfe Vitaglina nächst. Castelnuovo und bei der Kirche nächst Meligne (Juli bis zum Winter).

Grammitis leptophylla Sw. An schattigen alten Mauern um Porto Rosa; in Felsspalten in der Suttorina und auf den Abhängen des Monte Vermacz oberhalb Mulla bei Cattaro (März bis August).

Adianthum capillus Veneris L. Auf feuchten Felsen nächst dem Wasserfalle hinter Clissa, an einer Quelle gegenüber der Eremitage S. Girolamo am Marian bei Spalato, ferner in der Schlucht der Fiumera bei Cattaro (Mai bis November). 
Cheilanthes fragrans Hook. Auf einer Feldmaner rechts des Weges nach Fort Spagnuol oberhalb Castelnuovo (März bis zum Herbst).

\section{Ophioglosseen.}

Ophioglossum vulgatum L. In den Macchien (Meeresstrandgestrüpp) auf der Halbinsel Punta d'Ostro längs der dortigen Telegraphenleitung (April, Mai).

\section{Gramineen.}

Arrhenatherum elatius M. et K. Am Světi Juro des Biokovo auf Triften über $1000 \mathrm{~m}$. (Mai, Juni).

Holcus lanatus L. Auf der Marchesina gredda bei Clissa, auch im Wäldchen bei Castelnuovo und am Vermacz (Juni, Juli).

Andropogon Gryllus L. Auf sehr trockenen Hutweiden um Castelnuovo und am Bergrücken des Vermacz bis Trinità und Gorazda (Mai bis Juli).

Andropogon distachyos L. Auf Felsen längs des Meeresstrandes auf Punta d'Ostro (Juli bis September).

Andropogon hirtus L. Auf sonnigen Abhängen und Felsen nächst der Eremitage S. Girolamo am Marian, ferner hinter Fort Grippi bei Spalato; bei Stobretz, auf Punta d'Ostro und beim Pulvermagazin Kombur nächst Meligne (Juni, Juli).

Andropogon hirtus L. $\beta$. pubescens Vis. Ziemlich häufig auf Punta d'Ostro, zwischen Castelnuovo und Meligne, am Giovanni di Cattaro und am Vermacz bei Mulla (Juni, Juli).

Sacharum strictum Sprg. In Gräben im Zuppathale bei Cattaro (Juni bis August). Lagurus ovatus L. Auf der Spitze der Halbinsel Marian gegen Trau zu, auch auf Lesina, Punta d'Ostro und in der Suttorina (Mai bis Juli).

Polypogon monspeliense Desf. In salzhaltigen Gräben in der Suttorina und nächst dem Schlachthaus von Cattaro (Mai bis Juli).

Digitaria sanguinalis Scop. Auf Punta d'Ostro und längs des Uferweges von Cattaro nach Perzagno (Juli bis zum Herbst).

Tragus racemosus Desf. Auf steinigen uncultivirten Orten der Insel Lesina, bei Lapad, auf Punta d'Ostro, in und um Cattaro (Juni bis October).

Phalaris paradoxa L. In Getreidefeldern und Olivengärten auf Punta d'Ostro (Mai, Juni).

Alopecurus utriculatus Pers. Am Walle, auf dem Exercirplatze und nächst der Schwimmschule von Cattaro (Mai, Juni).

Crypsis schoenoides Lam. In einer ausgetrockneten Lache beim Fort Traste V (Juli, August).

Echinaria capitata Desf. Auf Felsen und Rainen an der Spitze der Landzunge Bodicelli bei Spalato (Mai bis Juli).

Agrostis olivetorum Godr. et Gren. Auf Grasplätzen zwisehen Castelnuovo und Meligne (Mai, Juni). 
Gastridium lendigerum Gaud. Auf Rainen und uncultivirten Orten in der Dobrota, am Giovanni di Cattaro und von Seagliari bis auf den Vermacz (Mai bis August).

Lasiagrostis Calamagrostis Link. In Gebüschen auf Punta d'Ostro (Juni, Juli). Phragmites communis L. Im Zuppathal bei Cattaro (Juli, August).

Arundo Pliniana Turr. An Wegrändern auf Punta d'Ostro (October).

Sesleria elongata Host. Auf Felsen oberhalb Castell Suzuracz und in Gebüschen bei Scagliari (Juni, Juli).

Sesleria temifolia Schrad. Auf Hutweiden am Vuči zub und auf der Biela gora in der Krivošie, 1000-1500 m (Juni, Juli).

Sesleria marginata Griseb. An felsigen Abhängen in der Krivošie, auf der Biela gora und am Monte Vermacz (Juni, Juli).

Koeleria cristata Pers. $\beta$. gracilis. Auf steinigen Hügeln um Spalato und an Abhängen oberhalb Mulla bei Cattaro (Mai, Juni).

Koeleria cristata Pers. $\gamma$. major. Auf Felsen am Monte Vermacz (Mai, Juni).

Koeleria phleoides Pers. An felsigen Abhängen um die Stadt Lesina, auf Punta d'Ostro, um Castelnuovo, Scagliari, am Wall von Cattaro und am Vermacz (Mai bis Juni).

Koeleria splendens Presl. Auf Hutweiden am Marian (Mai, Juni).

Poa pratensis L. Auf Wiesen und Hutweiden um Cattaro, auf Triften am Biokovo und in der Krivošie (April bis Juni):

Poa pratensis L. var. angustifolia. Am Giovanni di Cattaro (April, Mai).

Poa trivialis L. Auf Hügeln um Cattaro (April, Mai).

Eragrostis major Host. An Wegen um Ragusa, auf Lesina und Punta d'Ostro (Juli bis September).

Aira elegans Gaud. Auf sehr mageren Grasplätzen, zwischen Hecken um Spalato, auf Lesina, Punta d'Ostro, Castelnuovo und Cattaro bis auf den Vermaez (Mai, Juni).

Aira elegans Gaud. $\beta$. biaristata Godr. Auf Grasplätzen, Brachäckern, in Olivengärten auf Punta d'Ostro und um Castelnuovo (Mai, Juni).

Avena sterilis L. Auf Rainen, Hügeln und an Abhängen 1 m Spalato, Punta d'Ostro, Castelnuovo (Juni, Juli).

Avena barbata Brot. Am Wall von Cattaro, auf Hügeln um Trinità, aueh am Vermacz (Mai, Juni).

Avena sempervivens Alsch. Auf der Nordseite des Marian und längs des Weges von Spalato nach Salona (April bis Juni).

Melica ciliata Todaro var. Bourgaei Griseb. in Pantoczek. In Gebüschen auf Punta d'Ostro (April bis Juli).

Molinia serotina M. K. Auf unfruchtbaren Hügeln, sowie auf Steinpflaster in und um Spalato, auf Punta d'Ostro und um Cattaro (August bis October). Dactylis littoralis Willd. An felsigen Abhängen des Giovanni di Cattaro (Juni, Juli). Cynosurus cristatus L. Auf offenen Waldplätzen am Vermacz längs des Weges nach Trinità (Mai, Juni).

Festuca rubra L. Auf Triften am Biokovo bis $1500 m$ (Juni, Juli).

Z. B. Ges, B. XL. Abh. 
Scleropoa rigida Griseb. An Wegen, in Olivengärten um Spalato, Ragusa, auf Punta d'Ostro, in der Dobrota, am Giovanni di Cattaro bis auf den Vermacz (Mai bis Juli).

Vulpia ciliata Lk. Auf gepflasterten Wegen in und um Cattaro, bei Mulla und am Vermacz (Mai, Juni).

Vulpia Pseudo-Myurus Soy. Mit der vorigen besonders im Wäldehen am Vermacz (Mai, Juni).

Bromus arvensis L. Auf Aeckern um Spalato (Mai, Juni).

Bromus rigidus Roth. Am Giovanni di Cattaro (Mai, Juni).

Bromus molliformis Lloyd. Auf Grasplätzen um Cattaro, besonders am Giovanni di Cattaro und bei Seagliari (Mai, Juni).

Gaudinia fragilis Beauv. In Gebüschen längs des Meeres zwischen Castelnuovo und Meligne (Juni, Juli).

Triticum pinnatum Mönch. Auf den Abhängen des Vermacz oberhalb Mulla (Mai, Juni).

Agropyrum pungens R. et S. Auf Punta d'Ostro, von Mulla bis Perzagno (Juni bis August).

Agropyrum repens P. B. Zwischen Gestrüpp und in Hecken um Spalato und Cattaro (Mai bis August).

Lolium temulentum L. Auf Aeckern auf Punta d'Ostro (Mai, Juni).

Lolium temulentum var. robustum Rehb. Zwischen Getreide bei Toretta nächst Spalato, auch längs Wegen auf Punta d'Ostro (Mai, Juni).

Lolium subulatum Vis. Nächst dem Fort Prevlacea auf Punta d'Ostro (Mai, Juni). Hordeum bulbosum L. Auf Felsen und Abhängen des Monte Sella (Lověen) oberhalb Cattaro nächst der Militärschiessstätte bei Trinità bis nach Scagliari herab (Mai, Juni).

Aegilops triaristata Willd. Auf Hügeln und Abhängen bei St. Anna nächst Castelnuovo (Mai bis Juli).

Aegilops triuncialis var. glabra. An der Küste bei Spalato (Mai bis Juli).

\section{Cyperaceen.}

Carex Linkii Schrk. Auf Abhängen des Lověen (Monte Sella) an humusreichen Stellen bis Scagliari herab (April, Mai).

Carex nitida Host. Unter Gesträuche und auf Grasplätzen bei Trinità nächst Cattaro (Mai, Juni).

Carex verna Vill. Am Walle von Cattaro und im Zuppathale (März, April).

Carex muricata L. var. virens. An quelligen Orten, feuchten Aeckern in der Dobrota unter der Felsenwand ober Matteo (April bis Juni).

Cyperus flavescens L. In Gräben bei Cattaro (Juli bis September).

Cyperus fuscus L. Auf überschwemmten Wiesen und in ausgetrockneten Lachen längs der Salona gegen deren Ursprung zu (August, September).

Heleocharis palustris R. Br. Am Bache bei der Mühle von Kuti nächst Meligne (April bis Juni). 


\section{Juncaceen.}

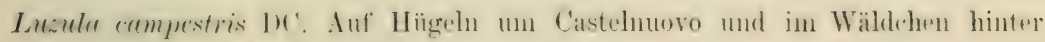
Seagliari (März bis Mai).

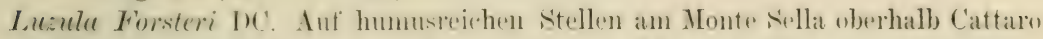
bis in das Wäldchen von Seagliari herab (März, April).

. Tuncus Tommasinii Parl. In Bratkwasser in der suttorina, auch bei T'eodo und Perzagno, sowie in der Dobrota (Mai, Juni).

. Juncus compressus . Jary. An sumptigen, quelligen Orten am Mereresufer beei Patludi nächst Spalato (Juni, Juli).

Funcus acutus I. Auf feurhten Wiesen bei s. ('ajo nächst Vrajnitza und an der Meeresbucht bei 'loretta nächst Spalato (Mai bis October).

\section{Colchicaceen.}

C'olchicum . Tunlace Freyn. Auf der Wiese längs der Strasse nach C'astell Suzurace bei S. Cajo gegeniiber von Vrajnitza (October).

('oleficum montanum L. Aut Puntal d'()stro, un Castelnuovo und bei dem Fort Traste (December bis März).

Colchicum Bironnae Vis. Auf Hutweiden beim Fort 'Traste IV und V (October). Colchicum autumnale I. Auf Grasplätzen am Monte Sella oberhalb Cattaro (im Herbst).

\section{Liliaceen.}

Tulipa silcestris L. Zwischen Felsspalten auf der Marchesina gredda bei Clissit (April, Mai).

Fritillaria montana Hoppe. In Wäldchen auf der Marchesina gredda mit der vorigen (März, April).

Fritillaria Meleagris L. Am selben Orte, auch zwischen Gesträuch auf Punta d'Ostro und um Cattaro (März, April).

Asphodelus albus Miller. Um Fort Traste (April, Mai).

Asphodelus luteus L. Häufig bei Mravinze, Salona, Spalato, Lesina und am Giovanni di Cattaro unterhalb der militär-optischen Telegraphenstation

S. Francesco (März bis Mai).

Ornithogalum narbonense L. An somigen Abhängen auf Punta d'Ostro (Mai, Juni).

Ornithogalum pyrenaicum L. Am Monte Marian, bei den Castelli und um Salonil, auf Lesina, Punta d'Ostro und am Giovanni di Cattaro (Mai, Juni).

Ornithogalum dicergens Borneau. Anf Ackererde auf der Südseite des Marian nächst der Eremitage und am Giovanni di Cattaro (Mai, Juni).

Ornithogalum comosum L. Cm spalato, Sitona und Clissal, sowie anf Punta d'Ostro (April, Mai).

O) mithogalum refiractum W. Krit. Auf der Südseite des Monte Marian (März, April).

Gragea arensis sichult. Auf Aeckern närhst den römischen sarcophagen bei Salona; auch zwischen Trinità und Cattaro (März, April). 
Scille autumnalis L. Am Monte Mossor, Marian, um C'astelnuovo, Scagliari und längs der Küste bis Budua (August bis October).

Scille bifolia L. In Gebüsche am Mossor, um Castehnovo, im Wäldchen bei Seagliari und am Vermacz (December bis Februar).

P'ancratium maritimum L. nach Visiani. In grosser Zahl am sandigen Meeresufer in der nördlichen Bucht von Traste gegen Cirtole zu (September, October).

Allium subhirsutum L. Zwischen Gestrüpp, in Hecken um Castelnuovo, anch längs der Strasse in der Dobrota (April, Mai).

Allium flaum L. Zwischen Gestein am Giovamni di Cattaro und oberhalb Scagliari (Juni, Juli).

Allium roseum L. $\beta$. bulbiferum. In Hecken, Wein- und Olivengärten auf Punta d'Ostro, um Castelnuovo, ferner am Walle von Cattaro bis in die Dobrota (April bis Juni).

Allium ursinum L. Im Walde am Monte Sella (Mai, Juni).

Allium paniculatum Alsch. Bei Castehuovo und Cattare (Mai bis Juli).

Allium rotundum $\mathrm{L}$. In verwilderten Weingärten auf Punta d'Ostro (Juni, Juli). Allium sphaerocephatum L. An steinigen Abhängen auf Punta d Ostro (Juni, Juli). Allium vineale L. Auf Brachäckern um Castelnuovo (Juni, Juli).

Allium longispathum Red. An Abhängen des Giovanni di Cattaro (Juli).

Allium fuscum Waldst. et Kit. An steinigen Abhängen um das Fort Gorazda (August).

Hyacinthus pallens Bieb. Auf Hutweiden auf der Marchesina gredda bei Clissia (April bis Juni).

\section{Amaryllideen.}

Sternbergiu lutea Ker. Auf den Felsen des Fort Mrare in Castelnuovo und nächst der griechischen Kirche von Topla (September, October).

Narcissus Tazetta L. Auf der kleinen Insel nächst dem Fort Arza am Eingang in die Bucht ron Cattaro, auch bei der Mü̈hle von Castelvecehio (April, Maai). Narcissus rudiiflorns Salisb. In den Macchien auf Punta d'Ostro (Februar, März).

\section{Irideen.}

Crocus vermus All. Auf Hügehn um Castehnovo und Meligne, an Abhängen des Monte Sella bis in das Wäldchen ron S'agliari, am Monte Vermacz bis Trinità und längs der Strasse nach Fort Traste V beim Dorfe Lessevice (Februar bis Mai).

Crocus reticulatus Stev. Am Monte Vermacz und in den Macchien bei Fort Traste VI (Februar, März).

Crocus dalmaticus Vis. Vermischt mit der vorigen (Februar, März).

Giladiolus triphyllus Sibth. Am Kamme des Monte Vermatcz links vom Fort in Nenge; auch bei Fort Traste VI (April bis Juni).

Iris Pseud-Acorus L. An Gewässern der Salona und am Bache bei der Mühle von Kuti (April, Mai). 


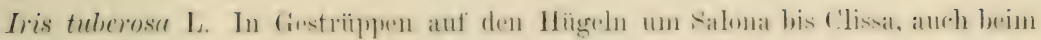
Fort Spagnuol oberhalb Castelnuovo (März, April).

\section{Orchideen.}

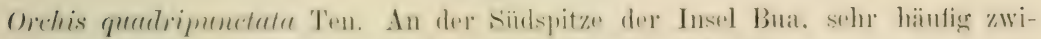
schen Punta d'Ostro und dem Dorfe Vitaglina (April).

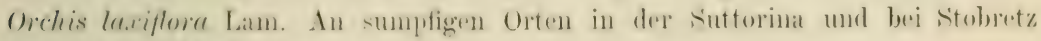
(April bis Juni).

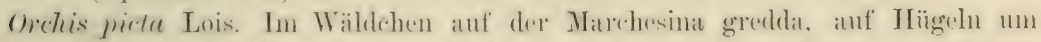
Salona, am Monte Vermacz und an der montenegrinischen neuen Strasse unter dem Fort Gorazda (April, Mai).

Orchis sambucina L. Auf Triften am Vuči zub in der Krivošie (Juni).

Orchis sambucina L. p. purpmere. An dem gleichen Orte in der Krivošie (Juni).

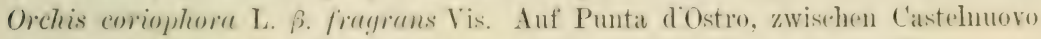
und Meligne; anch in der Zuppa und um Fort Traste (April, Mai).

Orchis prorinciulis Balh. fr. panciflora Vis. Im Wäldehen auf der Marehesinil gredelat, damn bei stolivo im Kastanienwalde und anf allen Abhängen des Vermacz (April, Mai).

Orchis tridentata seop). Auf der Insel Buil; anf der Nordseite des Monte Marian, auf der Marchesina gredda, sowie auf den Abhängen des Vermacz von T'rinità bis Perzagno (April bis Juni).

Orchis ustulata L. Auf Triften am Vuči zub in der Krivošie (Juni).

Anacamptis myramidalis Rirh. Aut Wiesen nürhst dem Fort Cerequizze in der Krivošie (Mai, Juni).

Anacamptis myramidalis Rich. var. condensata. Mit der vorigen am gleichen Standorte (Mai, Juni).

Gymmadenia conopsea Rehb. Auf Hutweiden beim Fort C'erequize (Mai, Juni). Spiranthes antumnalis Rich. Auf Grasplätzen, in Hecken und Olivengärten anf Punta d'Ostro, um Castelnuovo, bei Glavaty, endlich um Fort 'I'raste VI (August bis October).

Epripactis microplyylla sw. In Wäldchen zwisehen Castehnoro und Meligne. sorwie im Walde am Monte Vermacz (Juni).

Serapias cordigera L. Auf Grasplaitzen und in den Materhien aul' P'unta diostre, im Wäldchen bei s. Antonio zwischen C'astehnovo und Mteligne (Mai, Juni).

Serapias pseudocordigera Moric. In den Macehien $u$ das Pulvermagazin Kinmbur bei Meligne (Mai, Juni).

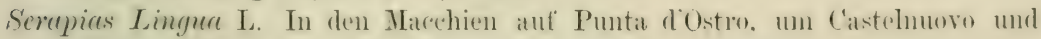
Meligne (Mai, Juni).

O) Jhrys cormuta ster. Auf der Nordseite des Marian, häutig im Wöldehen oher

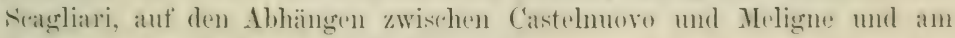
Vermaez ober Mulla (April, Mai).

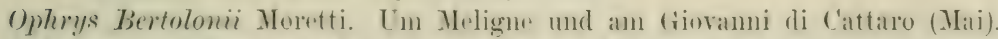

Ophrys atrata Lindl. Auf dex Nordseite ann Marian, hin und wieder aurh um Cattaro (April, Mai). 
Ophrys aranifera Huds. Am Giovamni di Cattaro (April, Mai).

Ophrys apifera Huds. In Olivengärten beim Fort Prevlacea auf Punta d'Ostro (April, Mai).

\section{Aroideen.}

Arisarum inlgare Targion. In humusreichen Felsspalten nächst dem Leuchtthurme auf Punta d'Ostro, an Felswänden in der Dobrota oberhalb Matteo, bei der Schiessstätte bei Trinità, in der Schlucht der Fiumera von Cattaro und in der nördlichen Bucht bei Traste (December bis Februar).

Arum Petteri schott. In Schluchten und Felsspalten am Lověen oberhalb Cattaro (Spigliari), auch ober Mulla und im Wäldchen am Kamme des Monte Vermacz (April, Mai).

Avum Dracunculus L. An felsigen Orten gleich hinter der Stadt Lesina längs der Strasse nach Cittavecchia (Mai bis Juli).

\section{Typhaceen.}

Typha angustifolic L. In Sumpfgräben im Zupluathale rechts von der Strasse nach Traste (Juni bis August).

Sparganium ramosum Huds. In sümpfen bei Stobretz und in Gräben nächst dem Militärspital zu Cattaro (Juni, Juli).

\section{Lemnaceen.}

Lemna minor L. In Strassengräben vor dem Militärspital zu Cattaro (April bis Juni).

\section{Potameen.}

Zanichellia pahustris Fries. In fliessenden Gewässern nächst der Mühle bei Kuti (Meligne) (April, Mai).

\section{Butomeen.}

Butomus umbellatus L. Im Cettinafluss bei Almissa (Juni, Juli).

\section{Alismaceen.}

Alisma Plantago L. In Gräben bei Stobretz (Mai bis Juli).

Alisma Plantago var. $\beta$. angustifolium. In Gräben um Cattaro (Juni, Juli).

\section{Coniferen.}

Pims halepensis Mill. Auf den Abhängen und Hügeln um Stobretz (Mai). Pimus Abies L. Auf dem Orien Wälder bildend (Mai).

\section{Cupuliferen.}

Fagus sileatica L. Am Orien, in der Krivošie, anf dem Radostak und der Dobrostitza bei Castelnuovo, sowie am Lovčen bei Cattaro (April bis Juni).

Castanea vesca Gärtı. Als Hain zwischen C'astehnovo und Meligne (Juni). 


\section{Euphorbiaceen.}

Andrache telephioides L. An der Stralsse nath Stobrety (April bis (october).

Euphortia platyphyllos I. B. literata. An sumptigen Uferrändern in der Suttorina (April bis Juni).

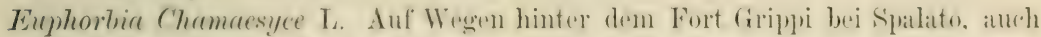
auf Punta d'Ostro und bei Cattaro (Juli bis September).

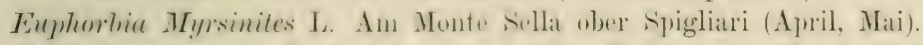

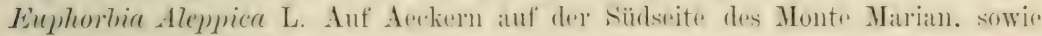
längs der Strasse nach Stobretz (Juli, August).

Euphorbia fragifera Jan. In Gebüsh auf der Marchesina greddla bei Clissit (April bis Juni).

Enphortia cappitulata Rehh). An Svèti Juro (des Biokovo) auf dessen hörhster Spitze, 5500' (Juni, Juli).

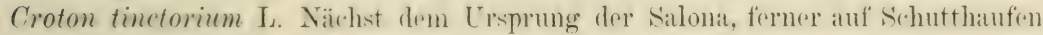
längs der neuen Strasse nach Trinità bei Cattaro (im Sommer).

\section{Aristolochieen.}

Aristolochia ('lematitis L. In Hecken. Weingärten und auf uncultivirten orten bei Clissat und längs: dem Wege von Castelnuovo nach MIeligne (Mïrz bis Mai). Aristolochia rotunda L. Zwischen Gestrüpp unter dem Fort Clissil, am Wrege von Glavaty nach Perzagno, in der Zuppa und um Cattaro (Mai bis Juli). Aristolochia pallida Wldst. et Kit. In Gestrüppen und Felsspalten am Honte Sellia an der neuen montenegrinischen Strasse (Mai, Juni).

\section{Cytineen.}

Cytimus Hypocistis I. Anf Wurzeln des Cistus satrifolius in den Maschien von Puntit dostro, ferner auf der Halbinsel Lustizza um dats Fort ('abballia und längs der Meeresküste daselbst (April bis Juni).

\section{Santalaceen.}

Thesium diearicatum Jan. Oberhalb des israelitischen Friedhofes bei Spalito. auch in den Macehien auf Punta d'Ostro (Mai bis Juli).

Osyris alba L. Um Castelnuovo und Cattaro. hier besonders am Gioramni di Cattaro (April bis Juni).

\section{Polygoneen.}

Polygonum maritimum L. Am sandigen Mreeresufer in der nördliehen Bucht ron Traste gegen Cartole zu (April bis October).

Polygonum dumetorum L. An Hecken auf Punta dOAstro (Juni bis September). Rumex tuberosus L. Unterhalls dem Fort Clissa gegen die Strasse zu, auf der Marchesina gredda, auf der Insel Bua, ferner bei der Militïrsehiessstitte bei Trinità nächst Cattaro (April bis Juni). 
Rumex multifidus L. Bei Meligne und Kombur und nächst dem Wäldchen am Kamme des Vermacz (Mai bis Juli).

\section{Chenopodeen.}

Chenopodium ambrosioides I. Auf Wegen und Manern auf Punta d'Ostro (Sommer bis zum Herbst).

Camphorosma monspeliaca L. Auf Felsen an der Meeresküste bei S. Stefano, Bodicelli und Paludi nächst Spalato (Juli bis November).

\section{Phytolaccaceen.}

Phytolacea decandra L. Zwischen Ruinen, an wïsten Orten nächst Häusern um Porto Rosa, Castelnuovo, Glavaty und in der Dobrota (Juli bis October).

\section{Amarantaceen.}

Amarantus prostratus Balb. Ueberall in Höfen, auf Schutthaufen und zwischen Steinpflaster in und um Spalato (Juni bis August).

\section{Plantagineen.}

Plantago Coronopus L. Längs dem Aufgang zum Fort Punta d'Ostro (Mai bis October).

Plantago Psyllium L. Auf Hügeln zwischen Salona und Mravinze (Mai, Juni). Plantago Bellardi Allion. An sehr mageren Abhängen längs des Kammes des Vermacz von Trinità bis zum Fort (April bis Juni).

\section{Plumbagineen.}

Statice incana L. Längs der Meereskïste auf der Nordseite des Monte Marian (Juli, August).

\section{Primulaceen.}

Primula acaulis L. Im Wäldehen zwischen Castelnuovo und Meligne, ferner am Monte Sella (Lověen) oberhalb Cattaro (Februar, März).

Primula suaceolens Bertol. Auf Waldblössen am Vuči zub in der Krivošie, 1500 m (April bis Juni).

Cyclamen repandum Sibth. et Sm. Im Gestrüppe der Ackerränder beim Dorfe Lessevice gegen Traste (März bis Mai).

Cyclamen neapolitanum Ten. Häufig auf den Felsen des Fort Mare von Castelnuovo, um Scagliari bei Cattaro, in den Macchien von Traste bis zum Dorfe Lessevice, auch in der Zuppa, bei Mulla und Perzagno (September bis November).

\section{Labiaten.}

Larandula Spica L. Auf der Nordseite des Monte Marian (Juni bis August). Rosmarinus officinalis L. In den Macehien von Punta d'Ostro, um Castelnuovo und längs der Küste bis Traste (Februar bis November). 
Salvia Horminum L. Auch auf Punta d'Ostro (Mai, Juni).

Salvia glutinosa L. In Wäldern am Lovčen oberhalb Cattaro (Juni, Juli).

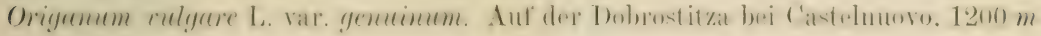
(September, October).

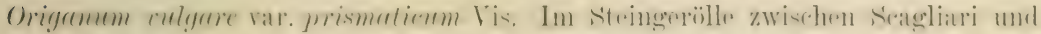
Cattaro (August bis Oetober).

T'hymus Zygis I. Auf dem Kamme der Dobrostitza (Juni, Juli).

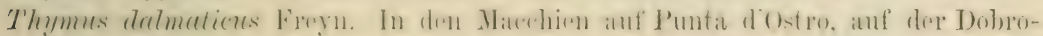
stitza und um Cattaro (Hai bis August).

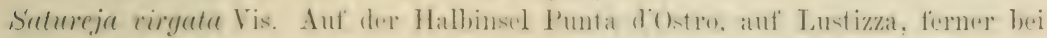

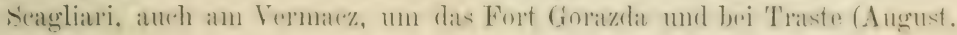
September).

Satureja montana L. e. communis. L'm ('lissa und sjalato, bei Castelnuovo, in der Dobrota, schr häufig bei Scagliari (August, September).

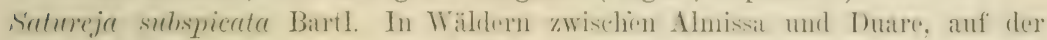
Dobrostitza nächt dem Dorfe Kameno (August, September).

Satureja hortensis L. Auf Brathen und Feldern unterhalb der römischen Wasserleitung bei Salona (Juli, August).

Nepela Calaria L. Auf der Dobrostitzal bei ('astelnuovo (Juni bic August).

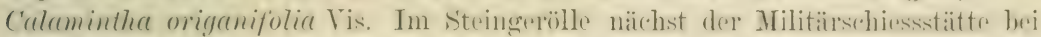
Triniti bis zur neuen montenegrinischen Strasse herab in grosser Anzahl (Juni bis August).

IIelissa officinalis L. B. rillosa Benth. Ant' wïsten Plätzon. nï̈chst Hänsern und in Hecken im Thale der Salonil, anch unterhalb des Fort Mare ron Castelnuovo (Mai bis August).

Clinopodium rulgare L. Auf Punta d'Ostro, um Castehnoro und Cattaro (Arai bis Juli).

Lamium hifectum Cyrill. In Hecken und zwischen Gerbüseh beim Dorfe Vitaglinat bei Castelnuovo, in den Anlagen vor der Stadt Cattaro, bei Scagliari und auf den Abhängen des Lovčen ober Spigliari (März, April).

Lamium maculatum L. Im Gesträuch links vom Fort Clissa und an Ackerrändern um Scagliari (März bis November).

Lamium intermedium Fries. Auf Brachen um Spalato (April, MIai).

Guleobdoton luteum Inds. Auf Waldpliatzen und in ILolzschlïgen an Biokoven und im Walde am Lověen oberhalb Cattaro (Juni, Juli).

Slachys spimelosa sibth. et sm. In Olivengiirten auf der Halbinsel Lalpad bei Gravosa (Mai bis Juli).

Stachys subcrenata Vis. Auf der Marehesina gredda und den umliegenden Bergen von Clissa, beim Dorfe Knezlač in der Krivošie (Juni bis August).

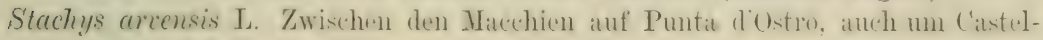
nuovo (Mai bis Juli).

Stachys recta L. An Ackerrändem beim Fort Trinita (Mati bis August).

Sideritis purpurea Talbot. Sehr häufig zwischen C'astehnoro und Mreligne (Mrai bis Juli).

Z. B. Ges, B, XI 。 Abl. 
Sideritis romana L. Bei Castelnuovo, am Yermacz ober Mulla bis Trinità (Mai bis Juli).

Ballota nigre «. foetida Koch. Auf wïsten Plätzen um das Fort Marre und auf den Stadtmauern von Castelnuovo, auch um Cattaro (Mai bis Juli).

Ballota rupestris Vis. Bei Salona, Spalato, Castelnuovo, auf Punta d'Ostro und nächst dem Fort Trinità (Juni, Juli).

Prasium majus L. In Felsspalten auf Punta d'Ostro (April bis Juli).

Ajuga reptans L. Bei Castehnovo, in der Dobrota, bei Gorazda und in der Zuppa (April, Mai).

Ajuga Chamaepitys Schreb. An Feldmanern unterhalb des Fort Grippi bei Spalato, auf Punta dOstro, um Cistelnuovo und auf der Insel S. Giorgio bei Perasto (März bis November).

Ajuga Chamaepitys forma glabrizscula Holuby. Auf Feldmauern unterhalb des Fort Grippi bei Borgo Lucacz (Mai bis Juli).

Ajuga Ira Schreb. Auf Punta d'Ostro (Mai, Juni).

Tencrium scordioides Schreb. In Hecken längs des Meeresstrandes bei S. Matteo in der Dobrota (Mai, Juni).

Teucrium flavum L. In den Macchien auf Punta d'Ostro (Juni, Juli).

Teucrium Arduini L. In der Schlucht der Fiumera ron Cattaro (Juni bis August).

Tencrium Chamaedrys L. var. acutilobum. Auf Felsen oberhalb S. Matteo (Mai bis August).

Teucrium Polium L. var. angustifolium. Sehr häufig in der Bocehe di Cattaro von Meligne angefangen bis zum Fort Gorazda und Trinità (Juni bis Norember).

\section{Rhinanthaceen.}

Euphrasia Odontites $\beta$. Bei Meligne, zwischen Seagliari und dem Militärspitil von Cattaro (August bis October).

Euphrasia Trixago Vis. In den Macehien auf Punta dOstro (Mai. Juni).

\section{Orobancheen.}

Orobanche minor Sutt. Um Castelnuovo, auf den Wällen der Festung Cattaro (Mai, Juni).

Orobanche crinita Vir. Auf den Abhängen links von der Fiumera di Cattaro oberhalb der dort befindlichen Häusergruppe (Juni, Juli).

Phelipaea ramosa C. A. Mey. Lm die Stadt Lesina gegen Cittarecchia, auf Punta d'Ostro und sehr häufig auf den Wällen und am Giovami di Cattaro (Mai, Juni).

Phelipaea larandulacen F. Sehultz. Mit der rorigen an gleichen Orten (Mai, Juni).

\section{Antirrhineen.}

Digitalis laevigata Wldst. et Fit. Im Thale ron Meligne unterhalb dem Fort Spagnuol (Juni bis August).

Linaria Cymbalaria Mill. ß. pilosa. Auf Felsen am Biokoro (Mai bis zum Herbst). 


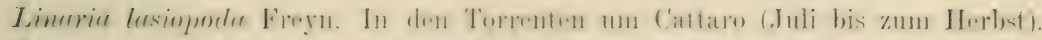

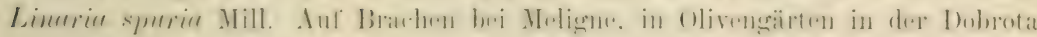
und im Steingerölle zwischen Seagliari und Cattaro sehr häufig (Juni bis August).

Linaria minor Desf. Im Schotter der Fimmera und bei Scagliari und auf dem montenegrinischen Bazar von Cattaro (Juni bis September).

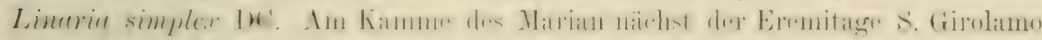
(April, Mai).

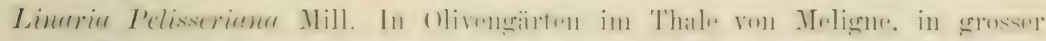
Zahl jedoch im Gebüseh links vom alten Fort Vermacz (Mai bis Juli).

Linaria chalepensis Mill. Oberhalb S. Matteo in der Dobrota (Mai, Juni).

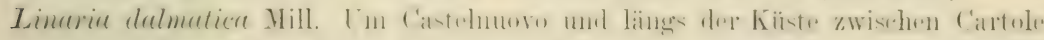
und Fort 'lraste VI (Juli bis October).

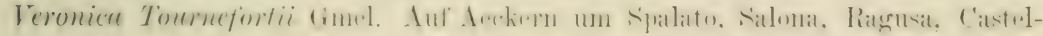
nuovo und bei Cattaro nächst dem Militärspital bis auf den Lovčen (März bis Juni).

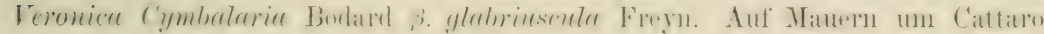
und auch sonst in Dalmatien (Februar bis April).

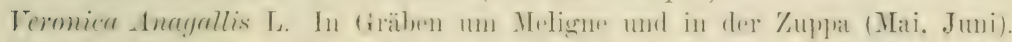

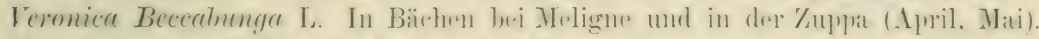

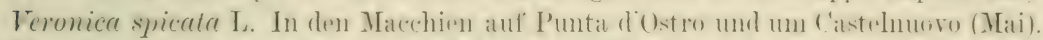

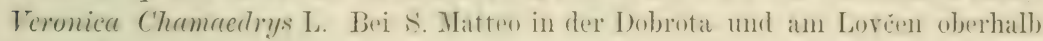
Cattaro (März bis Mai).

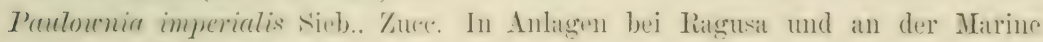
vor Cattaro (April).

\section{Verbasceen.}

Celsia orientretis L. An Abhängen zwisrehen der Marchesina greedda und Salona (April, Mai).

Terbuscum Lychnitis L. Auf der höchsten sitze der Ibobostitza bei Castelnuovo, $1200 m$ (Juli bis October).

Verbescum Thapsus I. Nïchst dem Dorfe Vitaglina am Monte Fobila und am Giovamni di Cattaro (Mai bis Juli).

Yerbuscum phoeniceum L. Auf der Insed Buat. auch in der Dobrota bei s. Matteo (April, Mai).

Terlusenm phlomoides L. An Abhängen des Nonte Kobila beim Dorfe Vitaglina (Mai bis Juli).

Verbascum floccostem W. et Kit. Auf Hëggeln bei s. Matten in der Dobrota (Mai bis Juli).

Serophularia peregrina L. Auf fenchten Feldmatuem um castehmoro und in der Dobrota (April, Mai).

\section{Solaneen.}

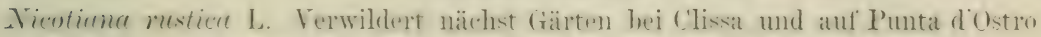
(Sommer bis zum Herbst). 
Solanum Duleamara L. Längs des Mecresufers bei Igalia bis nach Castelnuovo, auch am Wege von Mulla nach Perzagno (Juni bis October).

\section{Boragineen.}

Symphytum tuberosum L. Auf der Marchesina gredda bei Clissa (April bis August).

Symphytum bulbosum Śchimp. Links vom Wege von Mulla nach Perzagno (April bis August).

Cerinthe minor L. var. maculata. An Giovami di Cattaro (Mai bis August).

Echium plantargineum L. Bei Castelnuovo längs des Aufganges in die Stadt und bei Paludi (April bis Juni).

Lithospermum incrassatum Guss. Am Lověen bei der ersten montenegrinischen Ansiedlung oberhalb Cattaro (April bis Juni).

Lithospermum apulum Vahl. In der Dobrota, bei Scagliari und im Zuppathal (Mai bis Juli).

Lithospermum purpureocoeruleum L. In Hecken bei Salona und Clissa, auch bei Scagliari, in der Dobrota und Zuppa (April, Mai).

Lithospermum petraeum A. DC. Auf Felsen an Vermacz, oberhalb Mulla, am Giovanni di Cattaro und am Lověen (Mai bis Juli).

\section{Convolvulaceen.}

Convolvulus Cantabrica L. var. villifolius Gdgr. Auf Felsen um das Castell Grippi bei Spalato (Mai bis September).

Convolvulus sepium L. In Hecken und Gebüschen um Spalato, auf Lesinal, um Ragusa, auf Punta d'Ostro, bei Castelnuovo, Meligne und Cattaro (Juni bis September).

Cuscuta major. DC. Auf Toretta nächst Spalato (Juni, Juli).

Cuscuta Epithymum L. Auf dem Svèti Juro oberhalb Suzuracz hauptsächlich auf Salvia officinalis und Satureja (Juli bis October).

\section{Gentianeen.}

Gentiana nivalis L. Auf Triften am Vuěi zub des Orien nahe dem schmelzenden Schnee, 1500 m (Juni bis August).

Erythraea spicata Pers. Auf Wiesen rechts vom Ausfluss der Sillona längs dem Wege zum Bahnhof (August bis October).

\section{Apocyneen.}

Vinca minor L. Im Thal von Meligne im Wäldchen unterhalb dem Fort siprnunol (Mäi'z, April).

Nerium Olecunder L. Nächst dem Ursprung der Salona bei der römischen Wasserleitung; auf der Halbin:el Lapad beim Pulvermagazin daselbst; zahlreich auf Felsen bei Perasto (Juni bis August). 


\section{Asclepiadeen.}

Vincetoxicum Interi Vis, et Aschers. In den Macehien anf Punta d'Ostro, an

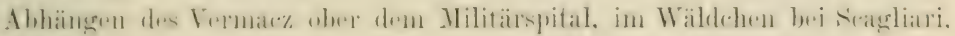
bei 'Trinitì und von dort nach Lepetane, auch sonst auf' den Abhängen des Lověen oberhalb Cattaro (April, Maii).

Cynunchum acutum L. lierhts ron der stratses, welehe ron Igala in die suttorina fïhrt (Juli, August).

\section{Ericineen.}

Erica raygus Buth. Auf' P’unta d'ostro, anf der Halbinsel Lustizza bis Burlua (Februar bis April).

\section{Campanulaceen.}

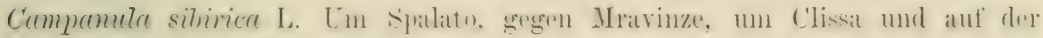
Dobrostitza (Mai, Juni).

Campanula glomerata L. Nächst dem Ursprung der Salona (Mai, Juni).

Campanula persicifotia L. Auf Waldplätzen am Biokovo (Mai, Juni).

Campanula bononiensis L. In der Suttorina (Juli, August).

Companula Reanunculus. L. Auch rom Punta dostro bis Cattaro (Mai bis August).

C'ampanula ramosissima sibth. Cmu C'astulnuovo, Mreligne und Perasto (Mai. Juni). Campanula capitata sims. Auf Puntal d'Ustro. gegen Meligne, $1 m$ Perzagno.

Mulla, in der Dobrota und bei Fort Traste VI (April, Mai).

C'ampanula falcate Roem. Auf Punta dostro und gegen Vitaglina (Mai, Juni).

\section{Ambrosiaceen.}

Ambrosia maritima I. An sandigen Meeresufer in der nördlichen Bucht ron Traste links vom Wege nach Cartole (Juli bis September).

\section{Compositen.}

Aster Tripotium L. An sumpitigen Meresgestade links vom Ursprumg der silonal, an Sümpfen bei Stobretz, auf der Insel S. Giorgio bei Perasto, auch um Mulla (September).

Erigeron acer L. Am Biokovo (Juni bis August).

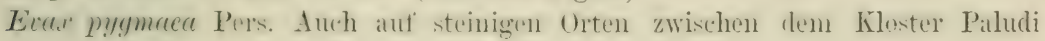
und 'loretta nächst Spalato (Mai, Juni).

Inuln Pritannicre L. Auf fenchten Arekern auf Lapad (Juli bis september).

Imula squarrosa L. Auch auf Punta d'Ostro (Juni, Juli).

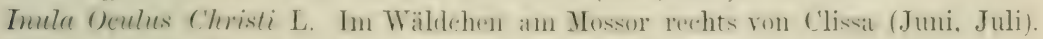
Inala montana L. Am Lověen oberhalb Cattaro (Juni, Juli).

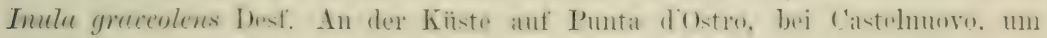
Cattaro bis Traste und Budua (August bis November).

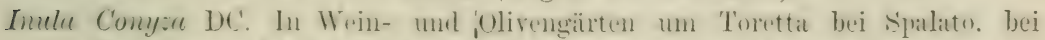
('astelnuovo. Mulla. Perzagno, Suggliari bis Traste (August, soptember). 


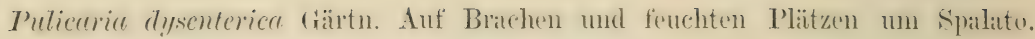
auf Punta d'Ostro und bei Castelnuovo (Juli bis September).

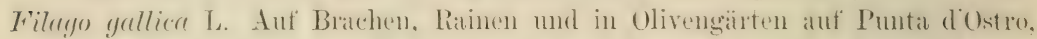

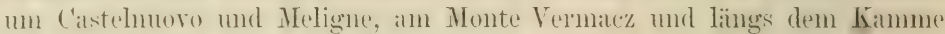
bis nach 'Trinità herab (Mai bis Juli).

Gnuphalium silraticum L. Auf der Dobrostitzal bei Castehuovo, $1500 \mathrm{~m}$ (Juni bis October).

Grunthalium luteo-ralbum L. Zwisehen Pflaster in Fort Clissa (August).

Artemisia coemlesens L. An Sïmpten bei Stobretz (September, October).

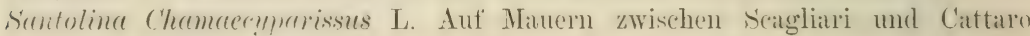
(Juni bis August).

Anthemis Pseudo-Cota Vis. An Gioramni di Cattaro nächst dem Pulvermagazin S. Francesco (Mai, Juni).

Anthemis Cotula L. Auf Punta d'Ostro und bei Kombur (Mai bis Juli).

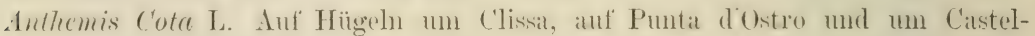
nuovo (Mai bis September).

Anthemis arensis L. 3. incrassala Boiss. In Acherrändern rechts am $\mathbb{W}^{\top}$ ege von Trinità nach Lepetane (Juni, Juli).

('hrysenthemum Lencenthemum L. Anf dem Abhange hinter dee Kirche s. Matteo in der Dobrota (Juni, Juli).

Pinurelia coronara Lees. Anf wïsten Plätzen an der Sitrasse von Lesinat natch Cittavecchia (Juni, Juli).

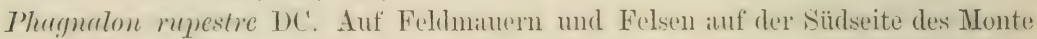
Marian (Mai bis Juli).

Senecio Visianiamus Papaf'. In Wäldernn der Krrivošie beim Fort C'erequizze (Juni bis September).

Senccio Cacaliaster Lam. Auf der hörhsten sjoitze der Dobrostitza ober Castelnuovo (August bis Oetober).

Echinops Ritro L. Längs der römischen Wasserleitung bei Salona bis gegren Mravinze und auf der Dobrostitza (Juli bis September).

Cirsium arense 3. restitum Koch. Auf Aeckern auf Punta dOstro (Juni bis August).

Cirsium palustre s.op. In sumptigen Gräben in Silona (Juni bis August).

Pycnomon Acanna Cassin. An Rainen um Clissa (Juni bis August).

Silybum marianum Gärtn. An sehr mageren Orten rechts ron der Strasse nach dem Omblathale, gleich ausserhalb Gravosa (Mai, Juni).

Tyrimmes lencographlus C'assin. Auf P'untal dOostro und anf den Abhängen des Vermacz gegen Trinità und Lepetane (Juni, Juli).

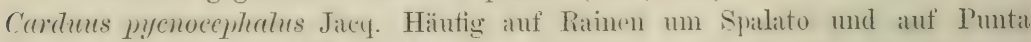
d'Ostro (Juni, Juli).

Chamaepence stellata DC'. Im Thale der C'ettina zwischem Almissa mol Duare nächst der dortigen Mühle (Juni bis August).

Carlina acaulis L. An Abhängen des Dtossor bei Dugopolje, anch gegenüber von Clissal unter der Felsenwand der Marchesina greddar (Juni bis August). 


\section{Dipsaceen.}

Dipsacus laciniatus L. An sumpfigen Orten zwischen Cattaro und Mulla (Juli bis September).

Succisa australis Rechb. Auf Sumpfwiesen bei Stobretz und in der Suttorina bei Castelnuovo (August, September).

Scabiosa suareolens Desf. Auf Hügeln um den Ursprung der Salona (August). Scabiosa gramuntia L. Auf der Dobrostitza, $1300 \mathrm{~m}$ (April bis October). Asterocephalus maritimus Sprg. In der Suttorina und bei Traste (August, September):

\section{Globularieen.}

Globularia cordifolia L. Am ()rien und auf den Abhängen des Lověen längss der nenen montenegrinischen Strasse von Cattaro (Juni, Juli).

\section{Valerianeen.}

Valerianella olitoria Möneh. Am Lovčen oberhalb von Cattaro über der montenegrinischen Grenze (April, Mai).

Talerianella hamata Bast. Auf Aeckern der Nordseite des Marian (April, Mai).

Fedia echinata Vahl. Auf der Südseite des Marian (April, Mai).

Fedia eriocarpa Roem. et Schult. Auf der Nordseite des Marian, an Abhängen des Vermacz bis Trinità und am Lovčen (April, Mai).

Centranthus ruber DC. Auf Manern um Spalato, bei der Mühle ron Topla bei Castelnuovo (Mai, Juni).

\section{Stellaten.}

Asperula cynanchica L. var. canescens Vis. In der Torrenta bei Seagliari (Juli, August).

Asperula arvensis L. Auf Aeckern nächst der Militïrschiessstïtte bei Trinità (April bis Juni).

Crucianella monspeliaca Vis. Auf Punta d'Ostro, auf der Halbinsel Lustizza, um Castelnuovo und auf den Abhängen des Vermacz bei Trinità (Mai bis Juli).

Rubia tinctorum L. In Hecken und an Wegen um Salona, auch im Zuppathale (Mai bis August).

Putoria calabrica Pers. Auf Felsen im Omblathale rechts von der Strasse zur Villa Caboga (Mai bis November).

Galium parisiense L. var. leiocarpum Tausch. Auf mageren Hutweiden um Castelnuovo und auf Caballa (Juni, Juli).

Galium parisiense L. var. trichocarpum Tausch. Auf Punta d'Ostro und am Vermacz (Juni, Juli).

Galium murale All. Auf Mauern, Stiegen bei Topla nächst Castelnuovo (Mai, Juni).

Galium verum $\beta$. trachyphyllum Wallr. In der Suttorina bei Castelnuovo (März bis August).

Galium silvaticum L. var. $\beta$. laevigatum. Im Wäldchen zwischen Castelnuovo und Meligne (Juli, August). 


\section{Caprifoliaceen.}

Lonicere implexa Ait. In den Miechien auf Punta d'Ostro (Mai, Juni).

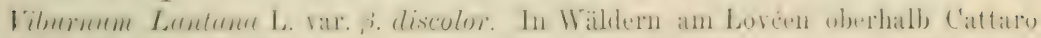
(IIii bis Juli).

\section{Umbelliferen.}

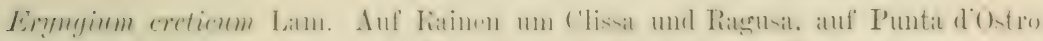
und längs der Küste bei Mulla und in der Dobrota (Mai bis Juli).

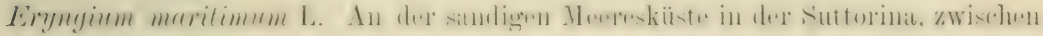
Perzagno und Stolivo und in der nördlichen Bucht von 'Traste unterhalb C'artole (Juni bis October).

Trinia vulgaris DC. Auf́ T'riften am Vuči zub in der Krivošie (Mai, Juni).

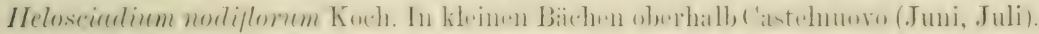

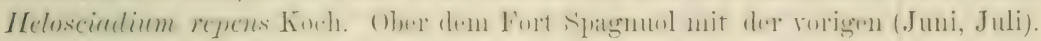
Ammi majus L. Auf Punta d'Ostro und um Castehnovo (Juni bis August).

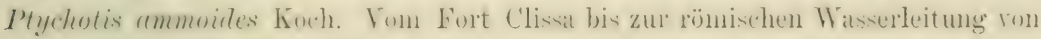
Salona (Mai bis Juli).

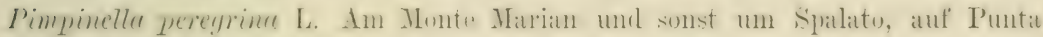
d'Ostro, um Castelnuovo und am Giovanni di Cattaro (Juni bis August).

Biasolettia tuberosa Fineh. Auf der Marchesinat greeddat und an Itorěen näthst der montenegrinischen Grenze (Mai bis Juli).

Bupleurum junceum L. Cm Silonil. Castelnuovo und auf Punta dOOstro (Juli bis September).

Oenanthe pimpinelloides L. Bei salona, in Gräben bei Castelnuoro und Cattaro (Mai bis Juli).

Oenanthe silaifolia M. B. Im 'Thale Begovina bei C'astelnuoro, auch bei Ciattaro (Mai bis Juli).

Sescli tomentosum Vis. Auf Felsen auf der südseite des Monte Marian beri der Eremitage S. Girolamo und bei Duare (August bis October).

Seseli montanum L. Bei Castehnovo, ober sciagliari und Mullat (september. October).

Sescli tortuosum L. An felsigen Orten bei Almissit (August bis Octuber).

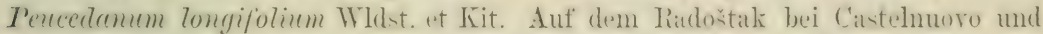
am Giovanni di Cattaro (August, September).

Pastinaca satica L. An Ritinen lïngs der Käiste bei Ifulla (Juni bis August). Heracten p'yrenaicum Pollin. Auf' Felsen und Hutweiden am Biokovo (Juni, Juli). Tordylium officinale L. Um Ragusa (April bis Juni).

7)ancars Carota L. 3. major Vis. Am Giovami di Cattare (August. Séptember).

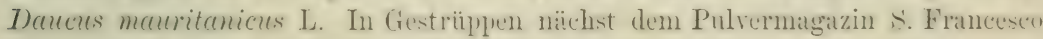
am Giovanni di Cattaro (Juni bis August).

Daneus Gingidium L. An wüsten Orten um Clissa und sigalate (ALit his Juli). Cancalis dancoides L. Lnter Getreide hinter dem Fort ('lissa (Mai bis Juli).

Echinophora spinosa L. In der Bucht ron Traste gegen Ciartole zu im Aeeressand, anch in der Suttorina (August, September).

7. B. Ges. B. XL, Abh. 
Smyrnium perfoliatum L. In Gebüschen und im Kastanienwäldchen von Stolivo, ferner am Lověen an der neuen montenegrinischen Strasse nächst der Grenze (Mai, Juni).

Smyrnium Olusatrum L. Auf wüsten Plätzen auf der Insel Buà (April bis Juni). Bifora testiculata DC. Beim Posthaus von Clissa und auf Aeckern nächst dem Sehlachthaus von Cattaro (Mai, Juni).

\section{Saxifragaceen.}

Saxifraga rotundifolia L. Im Kastanienwäldchen von Stolivo und am Giovanni di Cattaro (Juni, Juli).

\section{Crassulaceen.}

Sedum sexangulare L. Im Thale von Meligne (Mai, Juni).

Sedum Telephium \&. maximum L. Häufig am Giovanni di Cattaro (Juni bis October). Sedum glaucum Wldst. et Kit. Auf Punta d'Ostro und zwisehen Meligne und Kombur (Juni bis August).

Sedum neapolitanum Ten. Auf Felsen um Spalato, Meligne und auf Punta d'Ostro hinter dem Leuchtthurm (Juni, Juli).

Sectum album L. In Gebüschen zwischen Castelnuovo und Meligne (Juni, Juli). Sedum Cepaea L. Mit der vorigen (Juni, Juli).

Crassula ruberts L. Auf der alten Schiessstätte auf Punta d'Ostro (Mai, Juni).

\section{Paronychieen.}

Herniaria incana Lam. Zwischen Strassenpflaster im Fort Clissa (Mai bis Juli). Paronychia serpyllifolia DC. Auf den Bergen um Clissa, besonders längs der alten Marmont'schen Strasse und auf der Marchesina gredda (Juni, Juli).

\section{Cucurbitaceen.}

Bryonia dioica Jacq. Bei der Militärschiessstätte nächst Trinità bei Cattaro (Mai bis August).

\section{Tamariscineen.}

Tamarix africana Poir. Am Meeresufer nächst der Mühle zwischen Tran und Castell Stafileo, auf der Südseite des Monte Marian bei S. Stefano, in der Suttorina bei Castelnuovo und im Zuppathal bei Cattaro (Mai).

\section{Lythrarieen.}

Lythrum Hyssopifolia L. In Sümpfen um Stobretz, auch auf Punta d'Ostro (Juni bis August).

Lythrum Salicaria L. In Sümpfen bei Stobretz und in der Zuppa (Juni bis October).

\section{Callitrichineen.}

Callitriche stagnalis Scop. In fliessenden Wässern bei Castelnuovo (April bis October). 


\section{Granateen.}

Punica Granatum L. Auch in der Bocche di Cattaro von Punta d'Ostro bis Glivvaty und Mullat (Mai bis Juli).

\section{Pomaceen.}

Cotoneaster vulgaris Lindl. Auf Bergen um Castelnuovo (Miai, Juni).

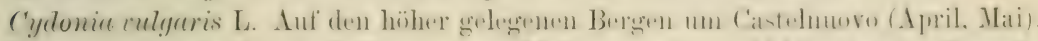

Pirus communis $\mathrm{l}$. Auf Bergen un Meligne und an . Abhïngen des Deresile oberhalb Bianca (April, Mai).

Sorbus domestica L. Lm sjalato, auf Puntal dostro und in der Dobrotal (April).

\section{Sanguisorbeen.}

Alchemille rulguris L. Auf Waldblösse'n am Orien und in der Krivosie (Juni, Juli). Poterium sprinosum L. Längs der Meereskiüste auf der Nordseite des Monte Marian gleich hinter den ersten Gärten (Mai).

\section{Rosaceen.}

Syirace Filipendula L. Auf der Marchesina gredda mol auf Punta dostro (Mai, Juni).

Fragaria elatior Ehrh. Auf Waldblössen um Cerequizze in der Kŕrivošie (Juni bis September).

Potentilla Tormentilla sibth. In Wäldern am Lověen oberhallb ('attaro (.Juni, Juli). Potentilla Fragariastrum Ehrh. In 'Thate und Wäldchen bei IIdigne und in Walde am Vermacz (März bis Mai).

Aremonia agrimonoides Neck. Am Vuči zub in der Krirossie (Mai, Juni).

Rosa alpina L. In Wäldern in der Krivošie (Juni).

Rosa spinosissima L. Bei Seagliari (Mai bis Juli).

Rosa canina L. Häufig in der Bocehe di Cattaro (Mai bis Juli).

Rosa scendens Mill. Auf Toretta bei spaliato, anch um Clissa, auf Punta dostro, dann auf den Festungsmauern von Castelnuovo und im Wäldchen bei Scagliari (Mai, Juni).

Rosa mubiginosa L. Um Castehnovo, in der Dobrota und bei Cattaro (Mai, Juni).

\section{Amygdaleen.}

Prumus Cerasus I. var. MIarasca Vis. Terwildert auf steinigen Abhängen um Cattaro (März, April).

Prumus Mahaleb L. Auf Hügehn in der Dobrota (März, April).

\section{Caesalpineen.}

Ceratonia Silique L. Auf Culturen und verwildert auf der Insel Buà, un Ragusat und auf Punta d'Ostro (im Herbst). 


\section{Papilionaceen.}

Genista tinctoria L. In Gebüschen um Castelnuoro (Juni).

Genista elatior Koch. In dem Wäldchen ober Castehnovo, besonder's aberhalb der Kirche von Topla (Mai, Juni).

Cytisus argenteus L. Längs des Gebirges oberhalb Castell Suzuracz, damn un Clissa, auch auf Punta d'Ostro (April bis Juni).

Cytisus capitatus Jacy. Auf der Marchesina gredda, bei Castelnuoro und Meligne (April bis Juni).

Cytisus infestus Guss. In den Macehien auf Lesina, um Ragusa und auf Punta d’Ostro (März, April).

Cytisus monspessulames L. Auf Punta d'Ostro und um das Pulvermagazin Kombur (März, April).

Cytisus spinescens Sieb. Auf Punta d'Ostro (März bis Mai).

Cytisus nigricans L. Bei Castelnuovo und Kombur (Juni bis September).

Cytisus Tommasinii Vis. Im Gestrüpp am Monte Vermacz zwischen dem Wäldchen dortselbst und dem Fort Trinità (Juni, Juli).

Cytisus Weldeni Vis. Längs den Felsenabhängen in der Dobrota oberhalb Matteo, ferner um Duare und auf Punta d'Ostro (Mai, Juni).

Lupinus hirsutus L. In Olivengärten anf Punta d'Ostro (April, Mrai).

Ononis Natrix L. Auf Hutweiden im Thal zwisehen Mreligne und dem Fort Spagnuol (Juni bis Angust).

Ononis Natrix rar. B. picta. In grosser Anzahl um die Stadt Lesina (Juni bis August).

Ononis Columnae All. Am Kamme des Marian ron der Eremitage S. Girolimo bis gegen den israelitischen Friedhof bei spalato (April bis August).

Ononis breviflora DC. Anf Grasplätzen nächst der Mühle zwischen Trau und Castell Staffileo.

Ononis reclinata L. Auf Punta d'Ostro (April, Mai).

Ononis antiquorum Koch. Auf Hutweiden bei Clissa, nächst der römischen Wasserleitung bei Salona; um Spalato, auf Puntal d'Ostro und in der Dobrota (Mai bis September).

Ononis repens Vis. Auf Brachäckern anf Puntal dostro (Juli bis Séptember).

Anthyllis Vulneraria L. var. pulchella. Am Rarlorštak und anf der Dobrostitzal bei Castelnuovo (Mai, Juni).

Medicago maculata W. Um Clissa, auf Punta d'Ostro, bei Castelnnovo und Cattaro (April bis Juni).

ITedicago coronata Desr. In Fort Clissa zwischen Steinpflaster (Mrai, Juni).

Meclicago Tispida Gärtn. (nach Freyn). Auf Puntal dOstro und Lesina, anch um Spalato und Cattaro (Mai, Juni).

IIedicago disciformis DC. Auf Hutweiden und Rainen um Ciattaro (Mai, Juni). Medicago falcata L. An Rainen bei S. Stefano und um das Fort Grippi bei Spalato (Juni bis zum Herbst).

ALedicago falcata p. versicolor Koch. Auf gleichen Orten mit der vorigen (Juni bis zum"Herbst). 
Medicargo Gerardi Wldst. et Kit. An Walle von Cattaro (Mai bis Juli).

Medicago litoralis Rohde. Am sindigen Meeresufer in der Bucht von l'raste gegen Cartole zи (Mai bis Juli).

Merticago marina L. Mit der vorigen im Meeressand (Mai, Juni).

Medicago orbicularis All. Auch um Castehnovo und Cattaro (Mai, Juni).

Medicargo sativa L. An Ackerrändern bei Clissal (Miti bis Juli).

Medicago tribuloides Desr. Im Fort Spagnuol auf Lesina (Mai bis Juli).

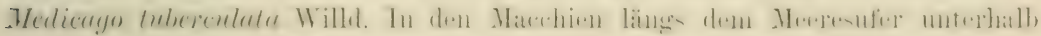
S. Antonio bei Castelnuovo.

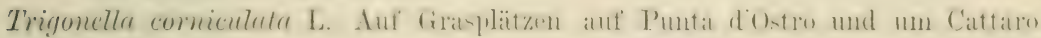
(Mai, Juni).

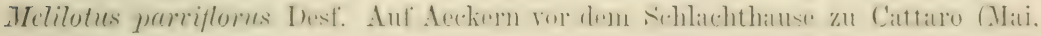
Juni).

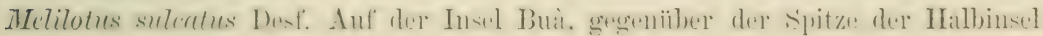
Marian (Mai, Juni).

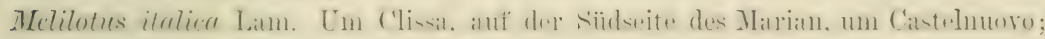
schr häufig auf den Abhängen des Giovanni di Cattaro (Mai, Juni).

Trifolium maritimum Huds. Bei Igala nächst Castelnuovo (Mai, Juni).

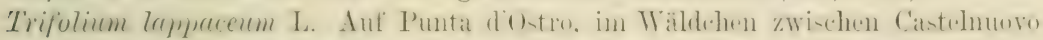
und Meligne, in der Dobrota und auf den Abhängen des Vermacz oberhalb Mulla und Perzagno (Mai bis Juli).

Trifotium diff̈usum Ehrh. Un Ciastrlunowo und anf dem Biokuro (Mai, Juni).

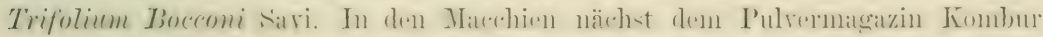
bei Meligne, ferner auf Waldblössen am Vermaez auf der gegen Scagliari zugewendeten Seite (Mai, Juni).

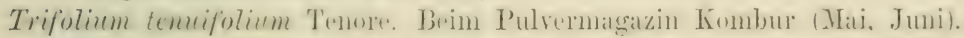

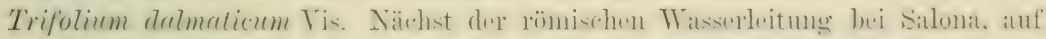
dem Friedhofe zu Grabovacz bei Zaguost, auf Punta d'Ostro und auf allen Bergen um Cattaro (Mai, Juni).

Trifolium Cherleri L. Aut Hutwriden anf der Nordseite des Monte Marian gleich hinter den ersten Weingärten (Mrai, Juni).

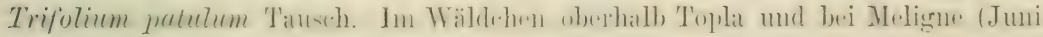
bis August).

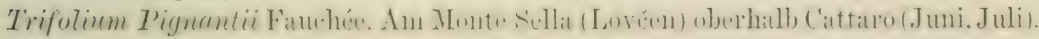

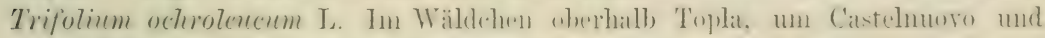
Meligne und am Monte Vermacz (Juni, Juli).

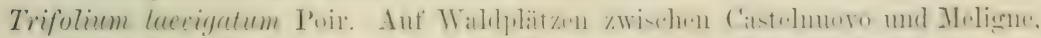
um den Militärfriedhof S. Antonio, häufiger im Wäldchen am Vermacz (Mai, Juni).

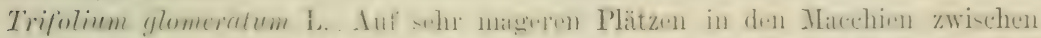

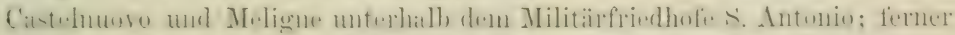
bei den Pulvermagazinen Kombur und Glavaty bis Perzagno (Juni, Juli).

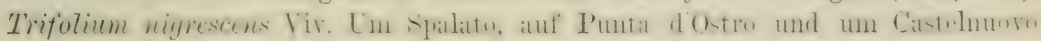
(April bis Juli). 
Trifolium subterraneum L. Auf Punta d'Ostro und um Castelnuovo, auch auf dem Devesile (April bis Juni).

Trifotium resupinatum L. An feuchten, sumpfigen Orten in der Suttorina und zwischen Castelnuovo und Meligne, bei Mulla und rechts von der Strasse zum Militärspital von Cattaro (April bis Juni).

Trifotium multistriatum Koch. Nächst dem Fort Prevlacea auf der Halbinsel Punta d'Ostro (Juni, Juli).

Trifotium patens Schreb. Auf Wiesen längs der Salona und links von der Strasse nach Trau (Juni, Juli).

Trifolium aureum Pull. Auf Wiesen um Fort Cerequizze in der Krivošie, auch bei Salona und längs der Strasse der Castelli (Juni, Juli).

Lotus edulis L. In Olivengärten auf Punta d'Ostro (April, Mai).

Lotus ornithopodioides L. In Olivengärten auf Punta d'Ostro, in den Macchien unter dem Militärfriedhof s'. Antonio bei Meligne, auch auf Lesina und bei Trinità nächst Cattaro (Mai, Juni).

Lotus cytisoides L. An felsigen Orten nächst der Küste auf Bodicelli und S. Stefano bei Spalato, um Ragusa, auf Punta d'Ostro, bei dem Inselfort Mamula, auch um Cattaro (Mai, Juni).

Lotus temifolius Reichbch. In Olivengärten auf Punta d'Ostro, in den Macchien zwischen Castelnuovo und Meligne (Juni bis August).

Lotus Dorycnium L. Auf Punta d'Ostro (Mai, Juni).

Hymenocarpus circinata Savi. Auf Hutweiden auf Punta dOstro, um Castelnuovo, ober Mulla bei Cattaro, in der Zuppa und auf den Abhängen des Vermacz bei Trinità (April, Mai).

Galega officinalis L. In beträchtlicher Menge um Castelnuovo (Mai bis Juli).

Astragalus sesameus L. Auf den der Eremitage S. Girolamo am Marian gegenüber liegenden Hügel und am Walle des Fort Grippi (April, Mai).

Astragalus virgatus Reichbch. Auf den Bergabhängen von Clissa bis Suzuracz, sowie auf der Insel Buà (April, Mai).

Astragalus hamosus L. An der Nordseite des Monte Marian hinter den ersten Weingärten (April bis Juni).

Scorpiurus subvillosa L. In Gebüschen auf Punta d'Ostro, um Castelnuovo und längs den Abhängen des Vermacz oberhalb Mulla bis Trinità (Mai, Juni).

Coronilla cretica L. Bei Castelnuovo, auf Punta d'Ostro, ober Mulla und Scagliari (Mai, Juni).

Coronilla stipularis Lam. Nächst der Eremitage S. Girolamo am Monte Marian (April, Mai).

Ornithopus compressus L. In Gebüschen beim Fort Prevlacca auf Punta d Ostro und im Wäldchen um den Militärfriedhof s. Antonio bei Castelnuovo (April bis Juni).

Hippocrepis comosa L. Auf den Hügeln nächst dem Ursprung der Salona (April bis Juni).

Hippocrepis citiata W. Gegen Mravince und Stobretz (Mai, Juni).

Hippocrepis unisiliquosa L. In Olivengärten auf Lapad (März, April). 
Onobrychis aequidentata D'Urv. Auch auf Punta d'Ostro (Mai, Juni).

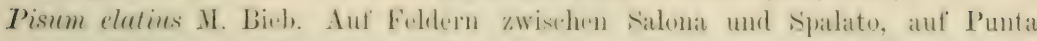
d'Ostro, um Castelnuovo, Perzagno, in der Dobrota, bei Seagliari; in grösserer Kahl jedoch am Giovamni di Cattaro (April, Mai).

Cicer arictimem L. In Culturen und rerwildert oberhall, Scagliari närhat der neuen montenegrinischen Strasse (Mai, Juni).

Errum nigricanes M. Bieb. Auf der Norelseite des Mente Marian (Mati. Juni).

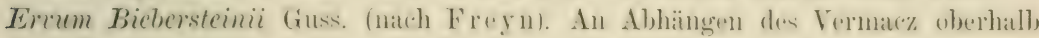
Mulla (Mai, Juni).

Ervum gracile DC. Auf Puntal d'Ostro (Mai, Juni).

Ticia peregrina L. Auf Puntal dostro, um Castelnuovo, in der Dobrotal, bei Scagliari, Mulla bis Gorazda (Mai, Juni).

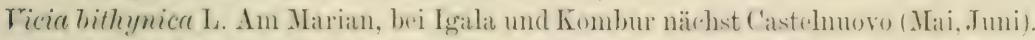

Ficia cassubica L. p. adriatica Fregn. Nächst dem Fort Prevlasea auf P'unta d'Ostro (Mai, Juni).

Ticia grandiflora seop. In Wäldehen um Dugopolje, bei C'lissa anf' P'unta diostro und um Castelnuovo (April bis Juni).

Ticia hirsula Koch. Auf Punta dOstro, bei Castelnuoro und Perzagno, sowie um Cattaro (April bis Juni).

Vicia hirsuta var. leiocarpa Vis. Vermischt mit der vorigen besonders um Ciattaro (April bis Juni).

Vicia hybrida L. Auf Punta d Ostro, um Castelnuoro und bei Cattaro (April his Juni).

Vicia tricolor Sebast. ot Maur. In Gestriippen und Hecken besonders um Clissá, auf Punta d'Ostro und weiters in der Bocche di Cattaro (April bis Juni).

Vicia ochrolenca Ten. Längs der montenegrinischen Strasse zwischen Scagliari und Trinità (April bis Juni).

Lathyrus ammus L. Bei Igala nächst Castelnuovo (Mai, Juni).

Lathyrus auriculatus Bertol. In Macchien bei Castelnuovo (Mai, Juni).

Lathyms Ochrus DC. Auf Aeckern vor dem Kloster Paludi, auch am Marian (Mai, Juni).

Lathyrus saxatitis Vis. Oberhalb dem israelitischen Friedhof am Marian bei spalato (April, Mai).

Lathyrus setifolius L. Am Marian, um Castehnovo, Perzagno, Mullal, am Giovanni di Cattaro und am Vermacz (April, Mai).

Lathyrus sphaerious Retz. Um das Fort Grippi bei Spalato und im Gestrïpp' auf Punta d'Ostro (April bis Juni).

Orobus albus I. Zwischen Felsritzen im Gestrüppe auf der Marchesina gredela. oberhalb S. Cajo bei Salona und ober Suzuracz (Mai, Juni).

Orobus variegatus T'en. In Gebüschen auf Punta dOstro, am Fermace und im Zuppathal unterhalb Trinità (April, Mai).

\section{Rhamneen.}

Rhammus Frangula. I. B. pumita. Anf' der Marchesina gredda bei C'lissit, in Ciebüschen ober Castehnoro, bei Scagliari und um Cattaro (Mai, Juni). 


\section{Polygaleen.}

Polygala amara L. Bei Cerequizze in der Krivošie und am Monte Sella bei Cattaro (April bis Juni).

\section{Violarieen.}

Viola silvestris Koch. Am Vuči zub in der Krivošie (Mai, Juni).

Viola lutea Sm. var. grandiflora. An Ackerrändern nächst der montenegrinischen

Niederlassung am Monte Sella ober Cattaro (Mai, Juni).

Viola tricolor L. var. arvensis. Am Světi Juro des Biokovo, in der Krivošie bei Cerequizze; auch am Monte Sella oberhalb Cattaro und um Spalato (Mai, Juni).

\section{Cistineen.}

Helianthemum arabicum Pers. Um Traste, auch am Vermacz gegen das Fort Trinità zu sehr häufig (Mai, Juni).

Cistus villosus L. Auf Punta d'Ostro, um Castehnovo, bei Traste, endlich am Kamme des Vermacz und an dessen Abhängen gegen Trinità und Lepetane (März bis Mai).

Cistus creticus L. Längs der Küste auf Punta d'Ostro und um Traste.

\section{Cruciferen.}

Matthiola simuta R. Br. In der sandigen Meeresbucht der Suttorina bei Castelnuovo (Juni, Juli).

Matthiola incana R. Br. Nächst dem Meere auf Punta d'Ostro, im Fort Spagnuol auf Lesina, auf den Festungsmanern in Castelnuovo (März, April).

Matthiola glandulosa Vis. In der nördlichen Sandbueht von Traste gegen Cartole zu (Juni, Juli).

Cheiranthus Cheiri L. Auf den Stadtmanern von Castelnuovo (März bis Mai). Arabis Turvita L. Am Vermacz und Monte Sella (April bis Juni).

Arabis hirsuta Scop. Auf der Marchesina gredda bei Clissa, um Castelnuovo und am Vermacz (April bis Juni).

Arcubis muralis Bertol. Am Vermacz oberhalb Mulla und am Monte Sella (Mai, Juni).

Arabis collina Ten. Mit der Vorigen an gleichen Orten (Mai, Juni).

Turritis glabra L. In der Dobrota bei Cattaro (Mai, Juni).

Cardamine maritima Portensch. Auf der Marchesina gredda bei Clissa; ober Mulla und längs der neuen Strasse im Wäldchen ober Scagliari (Juni, Juli). Hesperis glutinosa Vis. Auf felsigen Orten um Castelnuovo, auch häufig in der Dobrota und am Castell Giovanni di Cattaro (März, April).

Hesperis matronalis L. Längs der Strasse im Wäldchen ober Scagliari (Mai, Juni). Sisymbrizm Alliaria Scop. Am Monte Sella oberhalb Cattaro bei der ersten montenegrinischen Ansiedlung (April, Mai).

Sisymbrium Columnce Jacq. Am Giovanni di Cattaro (Mai bis Juli). 
Alyssum utriculutum sibth. Auf dem Vuéi zul, in der Krivošin; häufigre jerfoch

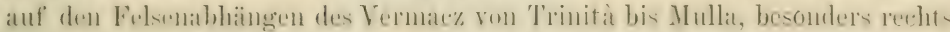
vom Aufgang zum Fort Vermacz (April bis Juni).

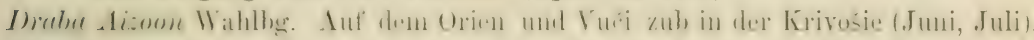
Thlaspi arense L. Hin und wieder in der Bocehe di Cattaro (Mai, Juni).

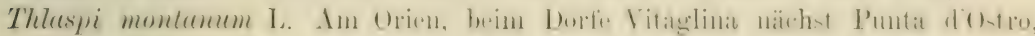
bei Castelnuovo und auf den Abhängen des Monte Sella (April, Miii).

Tberis serrulata Vis. Auf' Felsen und 'T'riften am Vuči zub (Mai, Juni).

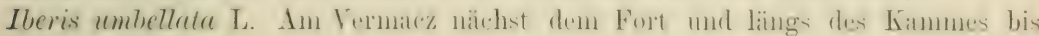
Trinitì (Mai, Juni).

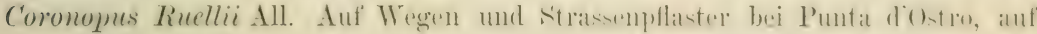
den Bankets und Wallgängen der Festung Cattaro und vor dem Fort Vermacz (Mai bis zum Herbst).

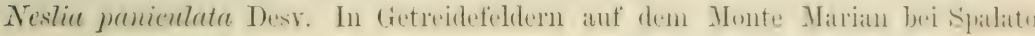
(April, Mai).

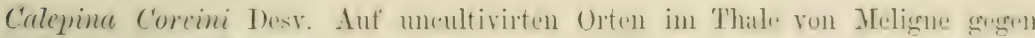
dis Dorf Kuti (April, Mai).

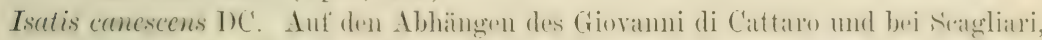
auch in der Dobrota (April, Mai).

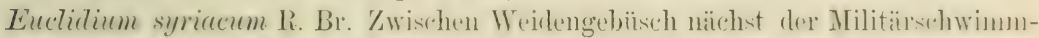
schule in Cattaro (Mai, Juni).

\section{Papaveraceen.}

Papaver apulum 'Ten. Hin und wieder im Gebiete (Mai bis August).

Peparer Rhoeas L. $\beta$. intermedium Freyn. Auf dem Giovamni di Cattaro (MLai bis August).

\section{Berberideen.}

Berberis rulgaris L. Auf fulsigen Orten in Wäldern un den Vuči zub in der Krivošie (Juni, Juli).

\section{Nymphaeaceen.}

Nymphaea alba L. In Sümpfen um Netkovich (im Sommer).

\section{Fumariaceen.}

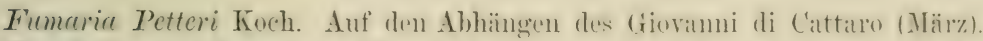

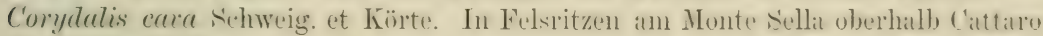
(April, Mai).

Corydalis solida Sm. Mit der vorigen am Monte Sella (April, Mai).

Corydalis ochrolenca Koch. Am sivèti Juro des Biokovo; am Mossor bei Dugropolje; auf der Marchesina gredda, schliesslich an Orien und am Monte Sella ober Cattaro (Mai, Juni). 


\section{Ranunculaceen.}

Delphtinium Consolida B. pubescens Freyn. In Brachfeldern und Olivengärten in der Dobrota, um Mulla und Perzagno (Juni bis November).

Delphinium Staphysagria L. und flore albo. In den Ruinen von Salona, im Fort Spagnnol auf Lesina, bei der griechischen Kirche ron Topla bei Castelnuovo und beim Fort Traste V (Mai bis Juli).

Helleborus multifidus Vis. Bei Radigne nächst Dizmo, am Schlossberg bei Sign und im Gebüsch links der Strasse von Sign nach Verlika (März).

Ranunculus Thora L. Auf Hutweiden nächst dem Vuči zub in der Krivošie (Juni, Juli).

Ranunculus parriflorus L. In ansgetrockneten Lachen beim Dorfe Viglina nächst Castelnuovo (März bis Juni).

Ranunculus muricatus L. Um Castehnuro und bei der Militärschwimmschule von Cattaro (Mai, Juni).

Ramunculus illyricus L. Auf der Insel Buà bei Trau (Mai, Juni).

Anemone coronaria L. An Ackerrändern und in Weingärten bei Spalato rechts von der Strasse nach Stobretz nächst der Villa Rhoitsch (Februar bis Mai).

Anemone apennina L. In Gestrüppen, Hecken und Gebüschen in der Suttorina, auf den Abhängen des Monte Sellia und Vermacz und unterhalb des Fort Gorazda an der montenegrinischen Strasse (April, Mai).

Thalictrum aquilegifolium L. Auf Wiesen beim Fort Cerequizze in der Krivošie (Mai, Juni). 


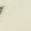



